\title{
Los upadres» pedagógicos de Europa. Discursos educativos fundacionales para la integración europea, cien años después de la Gran Guerra ${ }^{1}$ The pedagogical founding fathers of Europe: foundational education discourses for European integration, one hundred years after the First World War
}

\author{
Dra. Isabel VILAFRANCA MANGUÁN. Profesora Agregada. Universidad de Barcelona (ivilafranca@ub.edu). \\ Raquel CERCÓS RAICHS. Profesora Asociada. Universidad de Barcelona (rcercos@ub.edu). \\ Dr. Jordi GARCIIA FARRERO. Profesor Lector. Universidad de Barcelona (jgarciafub.edu).
}

\section{Resumen:}

El presente artículo navega desde las raíces fundacionales de la Unión Europea, cuya primera manifestación fue la «Europa de los Seis» ideada por los hoy denominados «padres de Europa», hasta los discursos educativos que abogaron por la instauración de la democracia en los países gestacionales de este acuerdo político supranacional. Al final de la Gran Guerra (1914-1918), de la cual se cumplen cien años, se inicia un proceso de reconstrucción pacífica en el que se establecen los cimientos de una federación europea para el mantenimiento de la paz interna. Europa, desde este punto de vista, no ha sido únicamente un ejemplo superlativo de entendimiento político, sino además, y principalmente, un éxito pedagógico. Por este motivo, Europa es, ha sido y será, ante todo, una vía pedagógica. Ante los nuevos retos que hoy se plantean -el desmembramiento a través del
Brexit, la desconfianza de los ciudadanos en sus instituciones, la constante amenaza para el mercado de las potencias emergentes, además de la realidad multicultural, por citar algunos-, la solución es necesariamente educativa. Los «padres» pedagógicos de Europa intentaron consolidar la democracia poniendo al ciudadano en el centro. Retomar y actualizar los discursos educativos fundacionales puede reorientar nuestra acción educativa para la reformulación de la Unión Europea.

Descriptores: Unión Europea, solidaridad internacional, educación cívica, educación para la paz, historia de la educación, educación europea.

\section{Abstract:}

This article starts by examining the roots of the European Union, the first manifestation

Fecha de recepción de la versión definitiva de este artículo: 15-02-2018.

Cómo citar este artículo: Vilafranca Manguán, I., Cercós Raichs, R. y García Farrero, J. (2018). Los «padres» pedagógicos de Europa. Discursos educativos fundacionales para la integración europea, cien años después de la Gran Guerra|The pedagogical founding fathers of Europe: foundational education discourses for European integration, one hundred years after the First World War. Revista Española de Pedagogía, 76 (270), 335-351. doi: 
of which was the «Europe of Six», conceived by a group of people nowadays known as the «founding fathers of the EU». It then moves on to consider the educational discourses that supported the establishment of democracy in countries where this supranational political agreement was developing. The end of the First World War (1914-1918), one hundred years ago this year, saw the start of a process of peaceful reconstruction in which the foundations of a European federation to maintain internal peace were laid. From this perspective, Europe has not only been a superlative example of political understanding but also, and predominantly, a pedagogical success story. This is why Europe is, has been, and will always be, a pedagogical project above all. In light of the new challenges facing it today (break-up as a result of Brexit, citizens' mistrust of its institutions, the constant threat in markets from emerging powers, and multicultural societies, to name but a few), the solution must be educational. The pedagogical «founding fathers» of Europe tried to consolidate democracy by putting the citizen at its centre. Returning to and updating its foundational educational discourses could reposition our educational actions for reformulating the European Union.

Keywords: European Union, international solidarity, civic education, peace education, history of education, European education.

\section{Introducción}

En el año 2012 la Unión Europea recibía el Premio Nobel de la Paz y, más recientemente, en 2017, era galardonada con el Premio Princesa de Asturias de la Concordia. Estos hechos conmemoraban un prolongado proceso, nada fácil, de reconstrucción pacífica. El viejo continente, con su cruento pasado como escenario de dos guerras mundiales, en el transcurso de estos cien años que ahora nos separan del final de la Gran Guerra (1914-1918), ha sido capaz de reestablecer sus relaciones políticas sin beligerancia. En esta compleja trayectoria, la educación ha tenido -y aún tendrá- un papel crucial. Frente a los primeros políticos que intentaron forjar un acuerdo intergubernamental -llamados «padres de Europa»-, se erigen una serie de discursos pedagógicos que acompañan la construcción de la ciudadanía europea 0, más bien, el intento de

afianzar la democracia parlamentaria que permite hacer de Europa una comunidad de convivencia pacífica. El presente artículo pretende ahondar en las figuras que hicieron posible, desde la Pedagogía, este proceso, destacando su decisivo papel en la configuración de sistemas educativos únicos y públicos.

\section{Los "padres de Europa». De la "Europa de los Seis» a la uUnión Eu- ropea»}

La construcción de la Unión Europea no ha sido ni un proceso exento de dificultades ni falto de encuentros y desencuentros. Si bien el impulso definitivo de esta gestación se da después de la II Guerra Mundial, ya antes - exactamente después de la Gran Guerra- había existido algún intento de reestablecer la paz a través del acuerdo intergubernamental 
de la creación de la Sociedad de Naciones en 1920, con sede en Ginebra, derivado de la aplicación del Tratado de Versalles. Inspirada en las ideas del presidente norteamericano Woodrow Wilson ${ }^{2}$, esta nueva organización con vocación mundial estuvo principalmente dominada por las potencias vencedoras europeas, Francia y Gran Bretaña.

En este contexto, coincidiendo con el periodo de entreguerras, y en la línea de forjar la integración europea e impedir nuevos enfrentamientos bélicos, cabe destacar la aportación de Richard N. Coudenhove-Kalergi que publica en 1923 en Viena la obra Paneuropa ${ }^{3}$. Formado en la capital cosmopolita de la república austríaca, este visionario arguye que la crisis del viejo continente -así como la pérdida de protagonismo de sus estados en el escenario mundial- no se debe a su avanzada edad biológica, sino política. La división política en veinte estados generaba, según Coudenhove-Kalergi, una anarquía internacional que debilitada la fraternidad entre ellos ${ }^{4}$. Lejos de una Europa sólida, fuerte, coherente y unida, este fenómeno provocaba el fin de la hegemonía europea en el plano internacional, frente al liderazgo mantenido durante el siglo XIX. Los primeros compases del siglo XX suponían el inicio del declive, sobre todo después del violentísimo enfrentamiento de la Gran Guerra derivada de los imperialismos y nacionalismos decimonónicos. Ante este ofuscamiento, para el austríaco la solución pasaba por establecer entre los países europeos una unión política que paliara la disgregación del momento. Y esta es, en definitiva, la idea central del título de su obra Paneuropa, que supone el primer proyecto moderno de una Europa Unida. Paneuropa es un concepto político de Europa en contraposición al geográfico. Para su autor, la frontera europea se extendía hasta donde llegaba el sistema político democrático. Su apuesta por esta unión política permanente era el remedio ante futuras guerras intraeuropeas, contiendas civiles al fin de cuentas. Si la fuente de los males era la división de sus estados, los nacionalismos constituían su principal patología. Los nacionalismos, a modo de religión secular, instauraban entre los estados fronteras infranqueables. A la luz de este diagnóstico, prescribía la separación entre estado y nación, destinada a disolver las fronteras nacionalistas respetando las minorías lingüísticas y culturales. Paneuropa pretendía ser una síntesis entre la nación europea, producto de la unidad de la cultura occidental, y la convivencia pacífica entre las diferentes tradiciones y minorías lingüístico-culturales. La idea de Coudenhove-Kalergi deviene un primer intento de entendimiento intergubernamental europeo cuando este no era más que una utopía soñada (Becerril, 2016), además de la única salida posible para mantener su rango en el contexto internacional. Por este motivo, entre otros, este autor ha sido considerado uno de los «padres de Europa».

No será hasta después de la Segunda Guerra Mundial cuando se renueve el afán de integración europea. Al término de la contienda, y dada la catastrófica situación en que el continente se encuentra, dividido, desacreditado y empobrecido, se impone la necesidad de propiciar el acercamiento político de los treinta estados soberanos, sorteando la anarquía e in- 
comprensión que había derivado en los dos conflictos armados. En este propósito tuvo mucha importancia la ilusión del que había ocupado el primer escaño de la Cámara de los Comunes de Reino Unido durante la guerra, Winston Churchill, que fundó en 1947 el «Movimiento de Europa Unida» (Churchill, 2016, pp. 78-87). Un año después de la fundación de este movimiento se realizará en La Haya el que se ha sido denominado "Congreso de Europa». Del 7 al 10 de mayo de 1948, en esta ciudad de los Países Bajos, tuvo lugar un encuentro inaugurado por la princesa Juliana y por el propio Churchill en el que se empiezan a construir las bases de la Unión Europea. Los asistentes y ponentes que lograron un acuerdo inicial son los considerados «padres de Europa»: Konrad Adenauer, Paul-Henri Spaak, Jean Monnet, Alcide de Gasperi, Robert Schuman y el ya citado Richard Coudenhove-Kalergi ${ }^{5}$. En el seno de la discusión de este primer congreso se hallan los desencuentros entre los «unionistas» - principalmente conformados por los representantes británicos, con el victoriano Churchill a la cabeza - y los «federalistas» - entre los que se hallan los franceses, italianos, belgas y neerlandeses-.

En el plano oficial, la consecuencia inmediata de este congreso es la creación de un Consejo de Europa, cuyo estatuto fue firmado en Londres en 1949. El Consejo de Europa, con sede en Estrasburgo, se compuso de dos organizaciones: un comité de ministros y una asamblea consultiva. Este Consejo tuvo un destacado rol en el plano europeísta. Y esto fue así porque proclamaba la unidad europea en base a «los principios de libertad individual, de libertad política y de preeminencia del derecho, sobre los que se funda toda democracia verdadera» (Truyol, 1972, p. 35).

Estos principios, que se consideraban el común denominador de la herencia compartida por los países europeos, eran los cimientos sobre los que establecer la naciente cooperación internacional del viejo continente. Precisamente, gracias al nacimiento el 4 de abril de 1949 de la Organización del Tratado del Atlántico Norte (OTAN) y dada la fragilidad institucional del Consejo de Europa, los europeístas decididos propugnaron una organización supranacional de ámbito geográfico más reducido, de comunidades europeas en sentido estricto, estableciendo en Ginebra el nuevo «Consejo de Municipios y Regiones de Europa» de 1951.

En el plano económico, los años siguientes a la Segunda Guerra Mundial fueron especialmente favorables. Entre 1945 y 1963 se habla de tres «milagros» económicos. Tres estados abanderan el crecimiento de Europa Occidental, Alemania, Italia y Francia. De los tres, el «milagro alemán» fue el primero y más decisivo. Bajo la dirección del canciller demócrata cristiano Konrad Adenauer (1945-1963) rápidamente Alemania occidental entró en bonanza económica. Gracias a una poderosa corriente de exportación y a una economía apoyada en una moneda sólida - deustche mark -, pronto se convierte en una de las primeras potencias del mundo. Aquella nación debilitada y devastada después de la guerra da paso a un estado opulento y envidiado, resurgiendo de sus cenizas como si de un ave fénix se tratara. Al «milagro alemán» le sucede el italiano, de naturaleza completamente 
distinta - y no tan acentuado como el primero-. Las regiones del norte y del centro se consolidan en sectores industriales como el automóvil y los electrodomésticos. Este progreso le asegura, durante veinte años, una posición pionera y fuerte en el mercado internacional. Al envite italiano le sigue el «milagro francés», que desde 1945 creció ininterrumpidamente hasta $1973^{6}$. Prácticamente treinta años gloriosos de imparable progreso hasta erigirse como la potencia hiperespecializada en la industria aeronáutica, agroalimentaria y biosanitaria que hoy es.

En realidad, esta coyuntura económica beneficiará la forja de una verdadera Europa. Aquí toman especial relevancia los llamados «padres de Europa». A medio camino entre la integración y la disociación, la actividad del Consejo de Europa se limitó a discusiones interesantes porque Reino Unido se opuso categóricamente a cualquier abandono de soberanía nacional. Tras este fallido arranque, Jean Monnet estimó que para realizar la construcción europea era preciso habituarse a trabajar juntos en el marco de realizaciones concretas en sectores precisos. $\mathrm{Al}$ hilo de su propuesta, Robert Schuman, ministro francés de Asuntos Exteriores -inspirado en la declaración de Monnet-, propone, el 9 de mayo de 1950, colocar el conjunto de la producción franco-alemana del carbón y del acero bajo una Alta Autoridad común. Se adhieren a la propuesta la República Federal Alemana, Bélgica, Italia, Luxemburgo y los Países Bajos. Así, el 18 de abril de 1951 - tras una breve negociación - se instaura la Comunidad Europea del Carbón y del Acero (CECA) cuya carta fundacional se recoge en el Tratado de París. Con el surgimiento de la CECA nace la «Europa de los Seis». Era la primera vez que los seis Estados miembros cedían ciertos derechos soberanos a un órgano común encargado de velar por los intereses de la nueva entidad. Esta Alta Autoridad, con poderes supranacionales, se ubicó en Luxemburgo. La CECA, cuya primera presidencia asumió Monnet, consiguió funcionar con gran éxito a pesar de la negativa de Reino Unido a participar en ella. A la vista de este triunfo, y tras el malogrado intento de instaurar una Comunidad Europea de Defensa, la idea de Europa fue relanzada por la reunión de los seis ministros de Asuntos Exteriores de la CECA en Mesina el 1 de julio de 1955. Al impulso que en este encuentro se le da a la $\mathrm{Co}_{-}$ munidad Económica Europea (CEE) se le ha dado en llamar la relance européenne. La realización material de la CEE será firmada en el Tratado de Roma del 25 de marzo de 1957, del que se han cumplido sesenta años. Por este motivo, el periodo que transcurre entre 1955 y 1957 ha sido denominado la «primavera europea». El objetivo de este acuerdo era garantizar la libre circulación de personas, bajo la protección de una ciudadanía común, y de mercancías diluyendo interna y paulatinamente las fronteras nacionales de los seis miembros. Al consejo de ministros heredado de la CECA se unía una Comisión Europea con sede en Bruselas. Era un primer bosquejo de poder supranacional (Carpentier y Lebrun, 1994).

Naturalmente, la creación de un mercado común europeo constituía un paso firme hacia la viabilidad de una Europa unida. En este proceso asume un papel 
fundamental el general De Gaulle que, sin ser contrario a la construcción europea, tal y como él relata en sus memorias, habría preferido un consorcio franco-alemán. De Gaulle advertía que la intromisión británica en los organismos de la CEE podía dejar la puerta entreabierta a la influencia americana ${ }^{7}$. Esta fue la principal causa del reiterado rechazo, al que se opuso en dos ocasiones (1963 y 1966), de la entrada de Reino Unido en el mercado común. Se trataba de fortalecer la Europa continental. La entente cordial protagonizada por De Gaulle y Adenauer pareció dar más importancia a la «consolidación» de la Comunidad que a la «ampliación» de sus miembros. Sobre todo, si este aumento podía poner en peligro la cohesión interna de los mismos frente al exterior, y en especial, frente a EEUU, a la luz de sus estrechas relaciones con la Commonwealth. Este hecho, así como la sempiterna mirada transatlántica del Imperio británico, incitó el veto de De Gaulle a la adhesión del Reino Unido. El general, De Gaulle proponía una «Europa de los estados», es decir, una Europa de la cooperación interestatal, de acuerdo con la idea de la nación-estado.

Ahora bien, las reiteradas solicitudes del Reino Unido, lideradas por el primer ministro laborista Harold Wilson para su incorporación a la Comunidad, provocaron intensas discusiones internas entre los seis integrantes. En el debate central las divergencias se dirimían entre la apuesta por una «Europa atlántica», de relaciones más centrífugas, o una «Europa europea», priorizando las concordancias centrípetas de la línea continental. Mientras De Gaulle objetaba la incorporación de Reino
Unido a la comunidad, otros, y en especial los declarados partidarios de la supranacionalidad -Luns y Spaak-, la apoyaban. Finalmente, tras duros y prolongados forcejeos en las cuestiones económicas -y en virtud de la desaparición de Churchill del panorama político-, la Cámara de los Comunes aprueba -el 28 de octubre de 1971 - la adherencia del Reino Unido a la Comunidad. Junto con la solicitud de dos candidatos más, el 22 de enero de 1972, la «Europa de los Seis» pasa en 1973 a constituirse como la «Europa de los Nueve». Se integran Reino Unido, Dinamarca e Irlanda. Este momento de ampliación consolida la segunda etapa en la construcción europea. La «Europa de los Pueblos» da paso a la «Europa de los Estados», de la que no tardará en comprobarse su insuficiencia. No será hasta 1981, con la unión de Grecia, que se hable de la «Europa de los Diez». En 1986, merced a la entrada de España y Portugal, se amplía la Comunidad denominándose, por entonces, la «Europa de los Doce». Y así sucesivamente, hasta llegar al Tratado de Maastrich de 1992 en el que se funda definitivamente la «Unión Europea» (Carpentier et al, 1994; Pérez Casado, 2017).

Recapitulando lo narrado, en esta extensa y complicada trayectoria se edifican los primeros cimientos de la Unión Europea, proceso protagonizado por las figuras de Richard Coudenhove-Kalergi - europeísta austríaco escritor de Paneroupa-, Jean Monnet - primer Presidente de la Alta Autoridad de la CECA-, Konrad Adenauer -Canciller alemán de la postguerra y promotor del milagro alemán-, Paul Henri Spaak - Presidente belga del partido socialista - y Robert Schuman - 
Ministro francés de Asuntos Exteriores, fundador e inspirador de la $\mathrm{CECA}-, \mathrm{Al}-$ cide De Gasperi -Presidente del Consejo de Ministros de Italia- y, como no, Charles De Gaulle - Presidente de la Quinta República Francesa cuyo nacimiento se produjo después de la crisis colonial de 1958-. Estos son los considerados los «padres de Europa».

\section{Los "padres» pedagógicos de Europa}

Frente a estos autores, que hicieron posible la construcción de la unidad Europea sobre la base de la convivencia pacífica, de la democracia y de la solidaridad intergubernamental, existe una pléyade de autores que, desde el discurso pedagógico, acompañaron este proceso de formación de los ciudadanos para la consolidación de estos acuerdos. Las tradiciones educativas de los países, en el momento de la fundación de la Europa de los seis, eran muy diferentes. De hecho, en la década de los veinte, tras el final de la Gran Guerra, en algunos países de Europa se instauró la democracia parlamentaria, mientras que en otros aún estaba por fundar. La democracia fue, sobre todo, cosa de tres países: Francia, Inglaterra y Alemania. En los dos primeros, la democracia no tuvo más que seguir la prolongada trayectoria que la guerra no había detenido (Carpentier et al., 1994). La herencia democrática, por tanto, estaba ya arraigada. No fue ese el caso de Alemania, donde la democracia estaba aún por constituir. Su realidad era otra y contaba con un límite inicial, derivado de su historia. Tras los fallidos intentos revolucionarios de los primeros meses, una Asamblea Constituyente elegida un año después del final de la Gran Guerra, en 1919, había fundado una república parlamentaria definida por la Constitución de Weimar que entró en vigor ese mismo agosto. Los primeros años de esta nueva república no estuvieron exentos de altercados, que no hacían más que denotar su fragilidad política. A los sucesivos intentos de golpes de estado de extrema izquierda y derecha, se añadieron asesinatos políticos, movimientos separatistas de Renania, la hiperinflación de la postguerra, el Putsch de Múnich protagonizado por Hitler en 1923 y la ocupación del Ruhr desde 1923 hasta 1925 por las tropas francesas y belgas. En cambio, a partir de 1925 el rumbo de la naciente república tomó nuevos derroteros, el país se estabilizó bajo la presidencia del Mariscal Paul von Hindenburg, que gobernó con una mayoría conservadora, y al que sucedió Hitler en 1933.

Simultáneamente a la instauración de la democracia en estos países, en la década de los veinte y los primeros años de los treinta, se extiende por Europa una ola que navega entre regímenes autoritarios 0 semiautoritarios hasta el extremo fascista adoptado por Mussolini en Italia después de la marcha de Roma (1922). Esta oleada aconteció principalmente por la Europa mediterránea y central, tras una breve experiencia de democracia parlamentaria. Así ocurrió en Hungría, bajo la dirección del Almirante Miklós Horthy (1920); en Austria, liderada por Monseñor Ignaz Seipel (1922); en España, por el General Primo de Rivera (1923); en Turquía, bajo el mando de Mustafá Kemal (1923); en Polonia, a cargo del Mariscal Józef Pilsudski (1926) y en Portugal, por António de Oliveira Salazar (1926). Se añade, a 
este complejo entramado político, la Revolución Soviética de 1917 y, sobretodo, la posterior Guerra Fría. Indudablemente, la oposición Europa-URSS durante este periodo aceleró la construcción europea dinamizándose, en consecuencia, la educación sobre la base democrática, contraria al comunismo. Precisamente, este fenómeno hace que los primeros «padres» pedagógicos de Europa se sitúen en los países de iniciativa democrática. Así pues, una primera generación de pedagogos, después de la Primera Guerra Mundial, y a la luz de los acontecimientos ocurridos, centra su discurso educativo en la formación cívica para la consolidación de la democracia en el estado. Sin embargo, tal y como veremos, sus propuestas superan estrictamente el orden nacional para entroncar al ciudadano con la humanidad en sentido genérico.

Aun a riesgo de olvidos, podríamos citar dos generaciones de «padres» pedagógicos de Europa:

Una primera generación que constituye la raíz de la construcción pacífica europea y que tiene la intención de consolidar la democracia a través de la autonomía del educando. En esta primera generación, cuya trayectoria se ubica principalmente en el periodo de entreguerras, se podría incluir a Kerschensteiner, Spranger y Natorp en Alemania; a Bovet, Claparède, Dottrens o Ferrière en Ginebra con su carga internacionalista; a Gloeckel en Austria; la reforma Viviani o el propio plan Langevin-Wallon de la segunda postguerra, en Francia.

Una vez solidificada la democracia en los países, se erigiría una segunda gene- ración de «padres» pedagógicos que, más allá de estas primeras iniciativas, después de la Segunda Guerra Mundial y tras la instauración de la CECA, luchan por el fomento de la unidad continental mediante el intercambio cultural de sus países, proclamando discursos en pro de la integración europea a través de la educación. Tal es el caso de Guido Gonella -Ministro de Instrucción Pública Italiano en tiempos de De Gasperi-, de Denis de Rougemont -Director del Centro Europeo de Cultura- y el impulso definitivo que Friedrich Schneider, pedagogo comparatista, ofrece en su obra la Educación europea, de 1963. Cabe mencionar, asimismo, una serie de instituciones que, al socaire de estos discursos pedagógicos, se instauraron con el afán de construir conjunta y cooperativamente la cultura europea, como por ejemplo el Centro Europeo de Cultura (1950) con sede en Ginebra 0 el Colegio de Europa (1950) de Brujas, además de la Asociación Europea de Educadores, con sede en Darmstadt, fundado en 1956.

Dentro de la primera oleada de iniciativas para la consolidación de la democracia, el ideal de re-construcción estatal pasa - casi por toda Europa en diferentes momentos- por la instauración de un modelo de educación única y pública que ya se había gestado antes de la Gran Guerra pero que se retoma con mayor ímpetu con posterioridad a ella. Afirma un autor que:

El movimiento de reforma de la enseñanza que había comenzado mucho antes de que estallase la guerra, se recrudeció durante esta, quizás por las mismas fallas que se revelaron en la preparación de los ciudadanos de los países beligerantes, fa- 
llas que se atribuyeron a deficiencias de los sistemas de enseñanza. Por eso, una de las primeras preocupaciones de la post-guerra ha sido la reforma y el mejoramiento de estos sistemas (La reforma escolar en Alemania, s.f., p. 7).

He aquí que, en el periodo de entreguerras, las reformas educativas se van sucediendo en el continente europeo. Se advierte que la reconstrucción del entendimiento intracontinental requiere de una nueva educación ${ }^{8}$. De ahí que hablemos de los primeros «padres» pedagógicos de Europa.

Intentaremos esclarecer quienes son estos primeros "padres» pedagógicos de Europa, para entender la función que ejercen en la forja de la democracia y la convivencia pacífica intra e interestatal. En realidad, la larga línea de pedagogos que en Alemania intenta instaurar la democracia se remonta a George Kerschensteiner, cuya aportación constituye la antesala de la reforma educativa que se gestará en la República de Weimar a partir de 1919. Según él, la acción educativa debe centrarse en la educación cívica, a fin de que el estado democrático devenga en una comunidad moral (1934, p. 33-34). Esta es la misión principal que debe adquirir la escuela pública y la educación en sentido genérico. No en balde, en la Alemania de la primera postguerra, la transformación se basará en la escuela del trabajo de Kerschensteiner. La reforma de la educación pública en Alemania hunde sus raíces en el movimiento de escuela unificada -Einhetsschule- cuyos principales defensores fueron los partidos democráticos y socialistas, así como las asociaciones de maestros y pedagogos de tendencia liberal entre los cuales se halla el propio Kerschensteiner, junto con Natorp, Fischer o Spranger, entre otros. La primera manifestación de este movimiento se hizo en junio de 1914 en la ciudad de Kiel, concretamente en la asamblea Deustche Lehvernien, en la que la asociación de maestros alemanes hizo suya las tesis de Kerschensteiner:

La escuela pública general en el estado jurídico, es decir, en aquel estado que regula automáticamente las relaciones de sus miembros según los principios de justicia, debe facilitar a todo niño, sin excepción, aquella educación a que tiene derecho en la medida de sus disposiciones (Luzuriaga, 1964, p. 105).

Dicho de otra forma, se fraguaba el interés por establecer en Alemania una escuela pública y democrática - proyecto que demorará el estallido de la Gran Guerra hasta la llegada de la república de 1919-. Junto a la aportación de Kerschensteiner, destaca el ideal de Pedagogía Social de Paul Natorp, que también sienta las bases de la democracia educativa desde una propuesta social, y la obra de Eduard Spranger, que propugna la finalidad educativa en la pedagogía cultural.

Si desde Alemania, dada la ausencia de tradición parlamentaria, la pedagogía se preocupa por establecer los cimientos de la democracia, desde Suiza, cuya capitalidad pedagógica asume Ginebra, el interés es otro: articular la mediación intergubernamental para el restablecimiento de la paz europea. Quizás la división interna de la población suiza y la adhesión dispar de sus ciudadanos -algunos partidarios de los franceses y otros 
germanófilos - hicieron que este estado se mantuviera neutral, tanto en la Primera como en la Segunda Guerra Mundial. Como imponían las condiciones del Tratado de Versalles, se instauró en Ginebra la Sociedad de Naciones en 1920 para liderar la reunificación europea. Sin embargo, fracasó en su intento por los intereses sucesivos de las potencias vencedoras. Pedagógicamente, Ginebra intentó liderar la reforma educativa a través de la divulgación del movimiento de Escuela Nueva y de la pedagogía activa. Pedagogos de la altura de Ferrière, Claparède, Bovet o Dottrens, entre otros, configuran la Escuela de Ginebra, que optó por el desarrollo de la pedagogía de corte experimental, sin desdeñar los ideales universalistas. El propio Adolphe Ferrière realizó un itinerario de iniciativas internacionalistas que van desde el Bureau International des Écoles Nouvelles (1899) o el Institut Rousseau (1912), hasta la fundación de la Escuela Internacional de Ginebra (1924-1950), pasando por la Liga Internacional de la Escuela Nueva (1921). Configuró, así, una teoría político-pedagógica que agrupaba los parámetros de europeísmo, internacionalismo, liberalismo, pacifismo, neutralidad, cooperación intelectual, puritanismo moral y confianza en la educación como forjadores de la sociedad futura. Como símbolo del espíritu de Ginebra, comparte la voluntad de una paz permanente basada en el desarme, en la cooperación internacional y en la voluntad de construcción de una utopía política y educativa (l'Ere Nouvelle) bajo los principios de autonomía moral e intelectual, y la actividad y el protagonismo del niño. Asimismo, Claparède y Bovet fueron los verdaderos motores del Institut Rous- seau. Además, Bovet estableció un observatorio internacional sobre el estado de la educación en el mundo con la fundación del Bureau International d'Education (BIE), que llegaría a constituirse como un órgano de documentación y promoción de los estudios científicos y objetivos en materia educativa. El BIE permitió el impulso y la consolidación de la pedagogía comparada como disciplina epistemológica, cognoscitiva y académica (Schneider, 1966), que posee una inequívoca vocación europeísta.

Todas estas iniciativas e instituciones, llevadas a cabo bajo el aliento del espíritu de Ginebra, trataban de establecer una unión europea en torno a los principios de comprensión, colaboración y cooperación internacional. En el periodo de entreguerras, y tras el desmembramiento de los imperios centrales, se trató de instaurar la paz a través del armamento moral, para lo cual la Escuela Nueva se consideró el medio más adecuado. El método educativo activo fue el escogido para hacer del niño un futuro ciudadano activo y participativo, a través del autogobierno, ideal de autonomía. En definitiva, se pretendía cimentar una ciudadanía europea que propiciara la paz perpetua en el continente a través de la enseñanza activa y del movimiento de Escuela Nueva.

Indiscutiblemente, la república austríaca no quedó exenta de este proceso reformista ${ }^{9}$ después de la caída del Imperio austrohúngaro acaecida como consecuencia de la Gran Guerra. En efecto, la decadencia cultural, social y económica de la última etapa de los Habsburgo obligó a la naciente república social-demócrata a rehacer una sociedad en plena crisis. Ahora 
bien, a partir de 1919, con la llegada al poder de los socialistas, se plantea cómo hacer de Austria un estado con una nueva estructura social acorde a la filosofía político-social del nuevo orden republicano. Al amparo de esta trasformación social -que impregnaba todos los ámbitos-, se proyectó una reforma educativa. Para ello, Otto Gloeckel asume la cartera de Subsecretario de Instrucción Pública (1919). Se forman 600 asociaciones de profesores para estudiar y practicar los principios de la reforma e introducirla en la república austríaca, implantándola en todas las regiones sin excepción. Los dos núcleos pedagógicos que la avalaron fueron: el principio de concentración, partiendo de los centros de interés; y el de irradiación, enseñanza global. Como puede deducirse, nuevamente la enseñanza activa toma prioridad en la instauración de la república y la formación del ciudadano. El objetivo era que el niño trabajase por sí mismo. No por azar, se cambió la Lernschule (escuela de aprendizaje), por la Arbeitschule (escuela del trabajo), sin desdeñar la educación cívica y moral, además de velar por la libertad religiosa, respetando la enseñanza católica, protestante y judía.

En el caso francés, el tema es diferente. Durante la Tercera República (1870-1940) se sucedieron una serie de transformaciones educativas que van desde la Reforma Viviani - a cargo de René Viviani, primer ministro pacifista de principios de la Gran Guerra-, hasta el plan Langevin-Wallon (1944-1945), inmediatamente anterior a la Cuarta República, cuyo presidente fue el ya mencionado Robert Schuman. El proyecto Viviani (1914) se inscribe dentro de las reformas de enseñanza unificada y de extensión de la obligatoriedad de la enseñanza técnica elemental, así como de la obligación post-escolar, además del principio de gratuidad de la escuela y del de democratización de la enseñanza media y superior, sin descuidarse la educación moral y del carácter. Este plan imponía una educación post-escolar obligatoria, profesional para los niños de trece a diecisiete años, y doméstica para las niñas, también desde los trece, pero únicamente hasta los dieciséis. En una línea similar, el plan Langevin-Wallon pretendía la democratización de la educación secundaria, a través de la enseñanza comprensiva. La solución era una escuela integrada, polivalente y comprensiva. Este ambicioso plan proyectaba una escuela única, laica, pero, sobre todo, que ofrezca una educación moral y cívica.

Al margen de estas iniciativas que apuntalaron la democracia desde la escuela, hubo una serie de personalidades destacables en el desarrollo del «espíritu europeo» desde la pedagogía. Nos referimos a la segunda generación de "padres» pedagógicos que, una vez consolidada la democracia a través de la educación, daba un paso adelante en pro del fomento de la ciudadanía específicamente europea desde las instituciones educativas. Cabe mencionar el ascenso en esta generación de la figura de Stefan Zweig, que, en 1932, insistía en la vía pedagógica para desintoxicar Europa de los males de la crisis de entreguerras, una crisis total, no únicamente económica, sino también epistemológica, política y filosófica, es decir, de sentido (Zweig, 2017, pp. 65-84). 
Habida cuenta de las evidentes tensiones intraeuropeas, Guido Gonella, Ministro de Educación italiano bajo la presidencia de De Gasperi, reclamaba un retorno a la unidad de la civilización europea sobre la base de su tradición. Gonella afirmaba las posibilidades de la unidad sin negar la pluralidad. No pretendía ni un intento de uniformidad ni de homogeneización cultural, sino reencontrar la tradición de Europa y su común civilización. En esta dirección advertía en un discurso de 1961:

La tradición europea es intelectual por la dignidad de un pensamiento común, es jurídica por el patrimonio de un derecho común, es espiritual por las afinidades internas de nuestra civilización común. [...] Volvamos pues a las fuentes de la civilización europea, fuentes que son tres: la griega, la romana y la cristiana. [...] La unidad de Europa se reconstruye restaurando, de entrada, el orden intelectual y social (Gonella, 1961, pp. 19-20).

Subyace aquí la aspiración de refundar pedagógica y humanísticamente Europa. Merced a este hecho, Gonella puede ser considerado uno de los «padres» pedagógicos de Europa de la segunda generación. Solo la vía cultural, y con ella la educativa, podrían salvaguardar la unidad europea. Consciente de las dificultades bélicas que había atravesado el continente, formulaba la urgencia de propugnar desde la escuela la solidaridad activa entre los ciudadanos y países. Solidaridad que había de basarse en una nueva justicia internacional que conciliara el concepto de nación con el de humanidad - tal y como habían aspirado muchos de los discursos pedagógicos de la primera generación- (Gonella, 1961, p. 21). Ante las nacientes fuer- zas antieuropeístas fascistas, había que buscar la paz con la democracia, arraigar el europeísmo desde el patriotismo.

Además de Gonella, mención especial merece, como «padre» pedagógico de Europa, Denis de Rougemont. Desde Ginebra, ya finalizada la Segunda Guerra Mundial, se impulsó el Centro Europeo de Cultura, que desde 1950 y dirigido por este convencido europeísta, fomentó el intercambio cultural destacando lo «común europeo» y reuniendo a personas y grupos que desempeñaban un trabajo similar. Esta institución educativa tuvo por finalidad «despertar el espíritu de solidaridad europea en las escuelas públicas, universidades y centros de enseñanza popular» (Schneider, 1963, p. 139). Al amparo de este centro, se creó la (AIEE) en 1951 que publicó una Bibliographie européenne, con la finalidad de divulgar los logros y problemas relativos a la unificación de Europa.

El ímpetu de Rougemont fue determinante para la integración pedagógica europea. Como Director del Centro Europeo de Cultura realizó una gran labor formando una élite de políticos y educadores europeístas. Consciente de que la unión aduanera no bastaba para el logro de una Europa unida, reclamó - desde el momento fundacional- una federación continental, política, social, cultural y económica. En sus escritos, subraya la importancia que concede a la educación para este entendimiento intergubernamental:

Decir que todo depende de la educación es decir que todo depende de los educadores y de su formación. El porvenir de Europa unida se juega en las Escuelas Normales. Mientras no se produzca un 
cambio de orientación antinacionalista y proeuropeo en ellas, y mientras este cambio no deje sentir sus efectos en la enseñanza secundaria de nuestros países, las bases mismas de la unión parecerán desarrollarse al paso que marquen políticos y economistas. Pues antes de «hacer Europa» hay que «hacerse de Europa». Y esto ocurre, fundamentalmente, en los espíritus: sin una «revolución cultural» previa no habrá ninguna revolución en las instituciones político-sociales, o no se pondrá realmente en marcha (Rougemont, 1975, p. 72).

En resumen, más allá de la Europa de los mercados, Rougemont hacía notar la necesidad de una Europa de los ciudadanos. Había que forjar una ciudadanía europea desde la educación. Primero, formando a los docentes en esta dirección, en las Escuelas Normales, para que, a modo de cascada, se dejara sentir posteriormente en la educación primaria y secundaria de cada uno de los países miembros. Rougemont era consciente de la necesidad de una «revolución cultural» para fortalecer el espíritu europeo. Solo la educación, y ningún otro medio, era el instrumento para lograr la unificación europea en la mente los ciudadanos. A la idea de Europa se tenía que llegar por la educación, despertando en cada persona un sentimiento de ciudadanía proeuropea, liberándose de los yugos nacionalistas que habían desembocado en las dos guerras intracontinentales.

Sin embargo, el envite decisivo de la educación europea, y por ello puede ser considerado otro de los «padres» pedagógicos de Europa, vino de manos de Friedrich Schneider, quien promulgó la necesidad de una disciplina llamada Educación europea que, además de ser el título de una de sus obras, constituía una propuesta curricular. La finalidad de la Educación Europea no era otra que la de:

Asegurar esta herencia europea -nosotros la designamos civilización europea o espíritu europe 0 - y darla a conocer en lo posible a muchos hombres de los pueblos de Europa; es decir, formar en ellos un sentimiento europeo, una conciencia europea, es una tarea eminentemente importante en la actualidad. Así pues, además de la integración política y económica, es necesaria una integración cultural que consolide las dos primeras (Schneider, 1961, p. 56).

Como ya recalcara previamente Rougemont, había que apuntalar la tradición europea a través de la educación, esto es, formar ciudadanos europeos. De este modo, propone un currículum educativo que contuviera, entre otros aspectos, una historia de la pedagogía en sentido europeo. Su intención era, como ya habían afirmado sus predecesores, alentar el europeísmo desde el patriotismo, siempre respetando los vínculos nacionales. Es así como debía configurarse una Educación Europea, cuyo sentimiento de cohesión no fuera disyuntivo con la comunidad nacional, sino compatible. Para ello, Schneider examinó los sistemas educativos europeos, destacando lo que tenían en común. Entre las características generales de los mismos halló la duración del tiempo escolar obligatorio - que oscilaba entre los seis y los diez años, logro que habían conseguido los «padres» pedagógicos de la primera generación-, la creencia en la educación permanente - más allá de la primaria y 
de la formación profesional, para los países europeos la educación se extendía a lo largo de la vida- y, por último, que en casi todos los países la organización escolar era ternaria - primera enseñanza, enseñanza media y educación superior-. Si los problemas educativos, tal y como él mismo descubriría, eran comunes, también la solución debía ser compartida por todos los estados miembros de esta unión. Por eso, la investigación pedagógica debía ser supranacional. En definitiva, todos los esfuerzos debían consagrarse a la búsqueda del espíritu europeo, tanto en sus problemas como en sus soluciones, ni más ni menos.

\section{A modo de conclusión}

Después del viaje realizado por los orígenes de Europa, no cabe duda de que esta se ha intentado articular, desde su gestación, como una vía pedagógica (Vilanou, Prats, Longares, 2016, p. 17). Acorde con la dimensión cultural y espiritual del viejo continente, Europa ha sido un modelo de referencia educativa, desde las propuestas alemanas y ginebrinas, hasta las austríacas y francesas, en favor de la construcción de un estado supranacional de convivencia pacífica y ciudadanía solidaria. Sin embargo, hoy se encuentra en una encrucijada. En el sexagésimo aniversario de su constitución, se enfrenta al desmembramiento de su unidad con la salida del Reino Unido y del inicio del proceso llamado Brexit, así como a sus posibles repercusiones en la eurozona. Anticipándose a su posible declive, Pérez Casado (2017) afirma que, ante la amenaza de los países emergentes y del competidor externo más potente, sobre todo después del triunfo en las urnas del America first, la alternativa es una más y mejor Europa. Se trata de repensarla soslayando el escollo tecnocrático, la pérdida generalizada de la confianza en sus instituciones y devolviendo el protagonismo a los ciudadanos. En esta misma dirección sostiene que «estas reformas en profundidad requieren la construcción de una pedagogía democrática que implique a la ciudadanía en los objetivos, que la convierta en agente cómplice de una nueva etapa en la construcción europea» (2017, p. 33). Así, la refundación de la Europa Unida o la Unión Europea, respetando y retomando los principios originarios de cooperación, pasan por la vía educativa, tal y como se lamentara años más tarde Jean Monnet: «Si tuviera que volver a empezar la construcción de Europa, lo haría por la cultura». Hoy pues, más que nunca, tiene sentido releer a los «padres» pedagógicos de Europa, los que instauraron la pedagogía democrática y la ciudadanía europea, permitiendo la posibilidad de la integración.

En esta nueva Europa que hoy nos encontramos, postindustrial, postmoderna, postorgánica y multicultural, el discurso pedagógico ha de tomar nuevas orientaciones para afrontar los retos que consoliden la reconciliación europea lograda a lo largo del periplo de estos cien años después de la Gran Guerra. Por eso, si algo es ahora Europa, o lo ha de ser, pasa por la vía pedagógica y educativa. Afirma Javier Solana que la Unión Europa es el proceso de integración más innovador y exitoso de la historia de la humanidad. (2015, p. 9 ). Si queremos mantener los niveles de prosperidad compartida, así como la libertad y la igualdad de los ciudadanos, la 
integración europea es imprescindible. La paz, la prosperidad, el Estado de derecho, el respeto a la diferencia, la solidaridad, la libertad, la democracia y la justicia social, valores que hoy conforman la identidad europea, que son nuestra «herencia común» y, como tal, queremos proteger, se han de afianzar por vía educativa. En fin, hoy más que nunca, Europa es una vía pedagógica.

\section{Notas}

1 La investigación que ha dado lugar a estos resulta. dos ha sido impulsada por RecerCaixa.

2 Afirma Voyenne que Wilson, asiduo lector de Kant, soñaba con el «Proyecto filosófico de la paz per. petua». La Sociedad de Naciones, de inspiración wilsoniana, constituyó el triunfo del derecho sobre los intentos beligerantes de hegemonía alemana en Europa. A saber, la victoria en Europa de la demo. cracia, de la justicia y de la paz sobre las aspiracio. nes de dominio bélico alemán. (1965, p. 165)

3 Previo a los intentos de construcción o reconstruc. ción europeas, después de la Gran Guerra se su. ceden una serie de obras denominadas «literatura de crisis». Un ejemplo es La decadencia de Occidente de Oswald Spengler, que denunció en 1918 la crisis del evolucionismo lineal y de los diseños progresivos del desarrollo social (Esquirol, 1994, p. 34).

4 Coudenhove-Kalergi (1923). Paneuropa. En Truyol, 1972, pp. 81-83. De Paneuropa hubo en 2010 una reedición en Madrid, publicado por Encuentro.

5 Afirma Voyenne con respecto a este congreso: «Por primera vez, todos los hombres decididos a edificar Europa se encontraban aunados. Resultó de todo ello una atmósfera de entusiasmo del todo excepcional. Sin embargo, después de este pri. mer contacto, se observó que dos tendencias, al menos, dividían gravemente las fuerzas europeas. Por una parte los «unionistas» permanecían tan re. sueltamente opuestos como en un pasado a todo lo que pudiera limitar la soberanía de los Estados [...]. Por otra parte, los federalistas, reclamaban una federación efectiva, dotada de poderes pro. pios» (1965, p. 188).
6 De Gaulle, en sus memorias, relata las sucesivas inversiones que durante el periodo que transcurre entre 1958 y 1962 se realizaron en Francia, desde el comercio, la agricultura y la electrónica, hasta la aeronáutica o las industrias derivadas del gas. Todas estas modernizaciones dieron un impulso definitivo a las regiones francesas, que se especia. lizaron en determinados sectores (1970, pp. 183. 184).

7 El propio De Gaulle cuenta en sus memorias las di. ficultades de construir una Europa europea, frente a la amenaza del Caballo de Troya atlántico que hu. biera supuesto la integración inicial de Reino Unido (1970, pp. 202-203).

8 No es de extrañar que en este periodo se consolide como disciplina científica la Educación Compara. da. Tal y como afirma Schneider «al término de la Primera Guerra Mundial, varias circunstancias favorecieron el desarrollo de la ciencia compara. tiva de la educación. Los estados con un sistema escolar desarrollado sintieron la necesidad de so. meterlo a un balance riguroso; los nuevos estados reconocieron en la educación el medio esencial de fundación y consolidación. Y, sobre todo, el afán de lograr una opinión y una humanidad cívicamente democráticas, condujo a ocuparse de planes de educación» (1966, p. 46).

9 Afirma Luzuriaga sobre la reforma educativa aus. tríaca: «La Primera Guerra Mundial vino a detener este desarrollo de la escuela nueva pública y solo a su terminación pudo ser proseguido. En tal senti. do, el segundo momento de esta es el de las 'insti. tuciones federales de educación' de Viena, que fue. ron verdaderas 'escuelas nuevas', pero de carácter público y nacional» (2002, p. 53).

\section{Referencias bibliográficas}

Anónimo (s.f.). La reforma escolar en Alemania. Madrid: Ediciones de La Lectura-Espasa Calpe.

Aguirre, M. (2017). Salto al vacío. Crisis y declive de los Estados Unidos. Barcelona: Icaria.

Adenauer, K. (2014). El final del nacionalismo. Madrid: Encuentro.

Becerril, B. (2016). Estudio introductorio Europe Unite. En W. Churchill, Europa Unida. Die- 
ciocho discursos y una carta (pp. 5-45). Madrid: Encuentro.

Cañellas, A., Solé, J., Pallach, J., Reventós, J., Trias, R. y Pujol, J. (1975). Les terceres vies a Europa. Barcelona: Nova Terra.

Carpentier, J. y Lebrun, F. (1994). Breve Historia de Europa. Madrid: Alianza.

Coudenhove-Kalergi, R. (2010). Pan-Europa. Madrid: Encuentro.

De Gaulle, C. (1970). Memorias de esperanza. La renovación (1958-1962). Madrid: Taurus.

De Gasperi, A. (2011). Europa, escritos y discursos. Madrid: Encuentro.

De Habsburgo, O. (2011). El camino de Europa. Madrid: Encuentro.

De Madariaga, S. (1951). Bosquejo de Europa. México: Hermes.

Esquirol, J. M. (1994). D’Europa als homes. Barcelona: Cruilla.

Ferro, M. (2014). La Gran Guerra (1914-1918). Madrid: Alianza.

Fichte, J. (1968). Discursos a la nación alemana. Madrid: Taurus.

Filho, L. (1936). La escuela nueva. Barcelona: Labor.

Freinet, C. (1975). El nacimiento de una pedagogía popular. Barcelona: Laia.

Fullat, O. (2005). L'Autèntic origen dels europeus. El cristianisme en la formació d'Occident. Barcelona: Pòrtic.

Gonella, G. (1961). Unitat d'Europa. Barcelona: Rafael Dalmau.

Güell, A. (Coord.) (1998). El Dilema de Europa. Barcelona: Ediciones del Bronce.

Howard, M. (2012). La Primera Guerra Mundial. Barcelona: Crítica.

Kamen, H. (1986). La sociedad europea (15001700). Madrid: Alianza.

Kerschensteiner, G. (1928). El alma del educador y el problema de la formación del maestro. Barcelona: Labor.
Kerschensteiner, G. (1934). La educación cívica. Barcelona: Labor.

Kerschensteiner, G. (s.f.). Concepto de escuela del trabajo. Madrid: La Lectura.

Luzuriaga, L. (1964). La escuela pública democrática. Buenos Aires: Losada.

Luzuriaga, L. (2002). La escuela nueva pública. Madrid: Losada.

Martín, V. (2015). Europa, un salto a lo desconocido. Madrid: Encuentro.

Natorp, P. (1915). Curso de Pedagogía. Madrid: La Lectura.

Negrín, O. y Vergara, J. (2003). Teorías e Instituciones contemporáneas de educación. Madrid: Centro de Estudios Ramón Areces.

Pérez Casado, R. (2017). La Unión Europea. Historia de un éxito tras las catástrofes del siglo $X X$. Valencia: Publicacions de la Universitat de València.

Rougemont, D. (1975). ;Europeos, uníos! Barcelona: Aymá.

Schneider, F. (1966). La Pedagogía Comparada. Barcelona: Herder.

Schneider, F. (1963). Educación europea. Barcelona: Herder.

Solana, J. (2015). Prólogo. En V. Martín, Europa, un salto a lo desconocido (pp. 9-10). Madrid: Encuentro.

Soler, J. (2008). Entre Europa i Amèrica Llatina: Adolf Ferrière i l'Escola Nova a Barcelona. Temps d'Educació, 35, 229-248.

Truyol, A. (1972). La integración europea. Madrid: Tecnos.

Vilanou, C., Prat, E. y Longares L. (2016). Entre la crónica i el compromís de futur. Temps d'Educació, 50, 11-29.

Voyenne, B. (1965). Historia de la idea europea. Barcelona: Labor.

Witte, E. y Backheuser, E. (1933). La escuela única. Barcelona: Labor.

Zweig, S. (2017). La desintoxicación moral de Europa y otros escritos políticos. Barcelona: Plataforma editorial. 


\section{Biografía de los autores}

Isabel Vilafranca Manguán es Doctora en Pedagogía por la Universidad de Barcelona y Profesora del Departamento de Teoría e Historia de la Educación de la Facultad de Educación de la Universidad de Barcelona. Se ha especializado en el estudio de las ideas y teorías pedagógicas, en especial las corrientes filosóficas y educativas vinculadas al idealismo alemán. Actualmente lidera el proyecto «Pensamiento pedagógico y discursos educativos en la construcción europea cien años después de la Gran guerra. Entre el pasado y el futuro», dentro del programa RecerCaixa.

Raquel Cercós Raichs es Licenciada en Pedagogía por la Universidad de Barcelona y Profesora asociada de la Facultad de Educación (UB). Se ha centrado en el estudio de los conceptos, discursos y narrativas de las Pedagogías de la Masculinidad a través de los vínculos que se establecen entre el poder, el género y la educación, fijando su punto de atención en la Inglaterra victoriana y su recepción y crítica en los Estados Unidos. Otra de sus líneas de investigación es la corporalidad y la pedagogía.

Jordi Garcia Farrero es Premio Extraordinario de Doctorado por la Universidad de Barcelona (curso 2012-2013), Profesor del Departamento de Teoría e Historia de la Educación de la Facultad de Educación de la Universidad de Barcelona y Consultor en el grado de Educación Social de la Universitat Oberta de Catalunya (UOC). Autor del libro Caminar. Experiencias y prácticas formativas (2014). 


\section{revista española de pedagogía año LXXVI, n 270, mayo-agosto 2018 \\ Spanish Journal of Pedagogy \\ year LXXVI, n. 270, May-August 2018 \\ sumario*}

table of contents **

\section{Estudios}

\section{Studies}

Xavier Úcar

Metáforas de la intervención socioeducativa:

implicaciones pedagógicas para la práctica

Metaphors for socio-educational intervention:

pedagogical implications for practice

Inmaculada Asensio Muñoz, Elvira Carpintero

Molina, Eva Expósito Casas y Esther López Martín ¿Cuánto oro hay entre la arena? Minería de datos con los resultados de España en PISA 2015

How much gold is in the sand? Data mining with

Spain's PISA 2015 results

Francisco José Fernández-Cruz, Ma José

Fernández-Díaz y Jesús Miguel Rodriguez-Mantilla

Diseño y validación de un instrumento

de medida del perfil de formación docente en

tecnologías de la información y comunicación

209
Design and validation of an instrument to measure teacher training profiles in information and communication technologies

\section{Notas}

Notes

Maria Teresa Caro Valverde,

María Isabel de Vicente-Yagüe Jara

y María Teresa Valverde González

Percepción docente sobre costumbres

metodológicas de argumentación informal

en el comentario de texto

Teacher perception of methodological habits

for informal argumentation in text commentary

Ana Rodriguez-Meirinhos y Esther Ciria-Barreiro

Revisión de intervenciones para mejorar

las habilidades pragmáticas en niños y

niñas con problemas de conducta y atención

Review of interventions to improve pragmatic language

skills in children with behaviour and attention problems

\footnotetext{
* Todos los artículos están publicados en inglés en la página web de la revista: www.revistadepedagogia.org.

** All the articles are published in English on the web page of the journal: www.revistadepedagogia.org.
} 
Pilar Pineda-Herrero, Anna Ciraso-Cali

y Mary Armijos-Yambay

Competencias para la empleabilidad

de los titulados en Pedagogía, Psicología

y Psicopedagogía: un estudio comparativo

entre empleadores y titulados

Employability and competences of Pedagogy,

Psychology, and Educational Psychology graduates:

A comparative study of employers and graduates

Isabel Vilafranca Manguán, Raquel Cercós Raichs y Jordi García Farrero

Los «padres» pedagógicos de Europa. Discursos educativos fundacionales para la integración europea, cien años después de la Gran Guerra

The pedagogical founding fathers of Europe: foundational education discourses for European integration, one hundred years after the First World War

Jonathan Ruiz-Jaramillo y Antonio Vargas-Yáñez

La enseñanza de las estructuras en el Grado de Arquitectura. Metodología e innovación docente a través de las TIC

Teaching structures on Architecture degrees.

ICT-based methodology and teaching innovation

\section{Reseñas bibliográficas}

Gargallo López, B. (Coord.) Enseñanza

centrada en el aprendizaje y diseño por

competencias en la Universidad.

Fundamentación, procedimientos y evidencias de aplicación e investigación

(Vicent Gozálvez). Ballester, L. y Colom, A.

Epistemologías de la complejidad y educación

(Carlos Alberto Pabón Meneses). Monarca, H.

y Thoilliez, B. (Coords.) La profesionalización

docente: debates y propuestas (Francisco Esteban

Bara). Balduzzi, E. Narrazione educativa e

generatività del perdono (Mauricio Bicocca).

373

313

\section{Informaciones}

XVI Conferencia Internacional de la Red

Internacional de Filósofos de la Educación 2018

sobre «Educación, diálogo y esperanza»;

ECER 2018 sobre «lnclusión y Exclusión,

¿Recursos para la Investigación Educativa?»;;

XV Congreso Internacional de Organización

335 de Instituciones Educativas (CIOIE) sobre

«Las tendencias nacionales e internacionales

en organización educativa: entre la estabilidad

y el cambio»;; Los ebooks monográficos de la

revista española de pedagogía.

Una visita a la hemeroteca (Gabriel Álvarez

López). Una visita a la red (David Reyero).

\section{Instrucciones para los autores}

Instructions for authors

Solicitud de originales

Call for papers

403

ISSN 0034-9461 - Depósito legal: M. 6.020 - 1958

e-ISSN 2174-0909 Rev. esp. pedagog. (Internet)

INDUSTRIA GRÁFICA ANZOS, S.L. Fuenlabrada - Madrid 


\section{The pedagogical founding fathers of Europe: foundational education discourses for European integration, one hundred years after the First World War ${ }^{1}$ \\ Los "padres" pedagógicos de Europa. \\ Discursos educativos fundacionales para la integración europea, cien años después de la Gran Guerra}

Isabel VILAFRANCA MANGUÁN, PhD. Associate Professor. Universidad de Barcelona (ivilafranca@ub.edu). Raquel CERCÓS RAICHS. Associate Professor. Universidad de Barcelona (rcercos@ub.edu). Jordi GARCÍA FARRERO, PhD. Assistant Professor. Universidad de Barcelona (jgarciaf@ub.edu).

\section{Abstract:}

This article starts by examining the roots of the European Union, the first manifestation of which was the "Europe of Six", conceived by a group of people nowadays known as the "founding fathers of the EU». It then moves on to consider the educational discourses that supported the establishment of democracy in countries where this supranational political agreement was developing. The end of the First World War (1914-1918), one hundred years ago this year, saw the start of a process of peaceful reconstruction in which the foundations of a European federation to maintain internal peace were laid. From this perspective, Europe has not only been a superlative example of political understanding but also, and predominantly, a pedagogical success story. This is why Europe is, has been, and will always be, a pedagogical project above all. In light of the new challeng- es we are facing today (break-up as a result of Brexit, citizens' mistrust of its institutions, the constant threat in markets from emerging powers, and multicultural societies, to name but a few), the solution must be educational. The pedagogical «founding fathers» of Europe tried to consolidate democracy by putting the citizen at its centre. Returning to and updating its foundational educational discourses could reposition our educational actions for reformulating the European Union.

Keywords: European Union, international solidarity, civic education, peace education, history of education, European education.

\section{Resumen:}

El presente artículo navega desde las raíces fundacionales de la Unión Europea,

This is the English version of an article originally printed in Spanish in issue 270 of the revista española de pedagogía. For this reason, the abbreviation EV has been added to the page numbers. Please, cite this article as follows:Vilafranca Manguán, I., Cercós Raichs, R., \& García Farrero, J. (2018). Los "padres» pedagógicos de Europa. Discursos educativos fundacionales para la integración europea, cien años después de la Gran Guerra | The pedagogical founding fathers of Europe: foundational education discourses for European integration, one hundred years after the First World War. Revista Española de Pedagogía, 76 (270), 335-351. doi: https:// doi.org/10.22550/REP76-2-2018-07 
cuya primera manifestación fue la «Europa de los Seis» ideada por los hoy denominados «padres de Europa», hasta los discursos educativos que abogaron por la instauración de la democracia en los países gestacionales de este acuerdo político supranacional. Al final de la Gran Guerra (1914-1918), de la cual se cumplen cien años, se inicia un proceso de reconstrucción pacífica en el que se establecen los cimientos de una federación europea para el mantenimiento de la paz interna. Europa, desde este punto de vista, no ha sido únicamente un ejemplo superlativo de entendimiento político, sino además, y principalmente, un éxito pedagógico. Por este motivo, Europa es, ha sido y será, ante todo, una vía pedagógica. Ante los nuevos retos que hoy se plantean -el desmembramiento a través del Brexit, la desconfianza de los ciudadanos en sus instituciones, la constante amenaza para el mercado de las potencias emergentes, además de la realidad multicultural, por citar algunos-, la solución es necesariamente educativa. Los «padres» pedagógicos de Europa intentaron consolidar la democracia poniendo al ciudadano en el centro. Retomar y actualizar los discursos educativos fundacionales puede reorientar nuestra acción educativa para la reformulación de la Unión Europea.

Descriptores: Unión Europea, solidaridad internacional, educación cívica, educación para la paz, historia de la educación, educación europea.

\section{Introduction}

In 2012 the European Union was awarded the Nobel Peace Prize and in 2017 it was awarded the Princess of Asturias Award for Concord. These awards commemorate a long and difficult process of peaceful reconstruction. The old continent, with its bloody past as the stage for two world wars, has, in the hundred years that separate us from the end of the First World War (1914-1918), been able to rebuild political relationships without belligerence. Education has played a crucial role in this complex journey and will continue to do so. In response to the first politicians who tried to forge an intergovernmental agreement - the so-called "founding fathers of Europe»- a series of pedagogical discourses were proposed to accompany the construction of European citizenship, or rather the attempt to strengthen parliamentary democracy to enable Europe to be a community of peaceful coexistence. This article offers an in-depth examination of the people who, through pedagogy, made this process possible, drawing attention to their decisive role in establishing unitary public education systems.

\section{The ufounding fathers of Europen: from the "Europe of Six" to the "European Union»}

The process of construction of the European Union was not without diffculties or disagreement and arguments. While the final push for this development came after the Second World War, before then -in fact, after the First World War - there had been an attempt to restore peace through the intergovern- 
mental agreement to create the League of Nations in 1920. This Geneva-based organisation was a result of the application of the Treaty of Versailles. Inspired by the ideas of the Woodrow Wilson', the president of the USA, this new organisation with its global mission was principally dominated by the victorious European powers of France and Great Britain.

In this context, coinciding with the interwar period, and in relation to forging European integration and preventing new armed confrontations, it is worth noting the contribution by Richard N. Coudenhove-Kalergi who published the work Pan-Europa in Vienna ${ }^{3}$ in 1923. Raised in the cosmopolitan capital of the Republic of Austria, this visionary argued that the old continent's crisis and the waning importance of its states on the world stage were not due to an advanced biological age but rather politics. According to Coudenhove-Kalergi, the division into twenty different states created an international anarchy that undermined the fraternity between them ${ }^{4}$. Far from leading to a solid, strong, coherent, and united Europe, this phenomenon was the cause of the end of European hegemony in the international sphere, in contrast with its position of leadership in the nineteenth century. The early years of the twentieth century saw the start of this decline, above all after the extremely violent clash of the First World War which resulted from nineteenth-century imperialisms and nationalisms. Faced with this dimming, for the Austrian author, the solution was to establish a political union among European countries to alleviate the breakup of that time. This is, essentially, the central idea behind the title of his work Pan-Europe, which is the first modern project for a united Europe. Pan-Europe is a political concept of Europe instead of a geographic one. For its author, Europe's frontiers extended as far as the democratic political system. His focus on this permanent political union was intended to be the answer to future intra-European wars, which were civil wars at the end of the day. If the source of Europe's evils was its division into states, nationalism was its main pathology. Nationalism, as a sort of secular religion, established impassable borders between states. In light of this diagnosis, he prescribed a separation of state and nation intended to dissolve nationalist borders while respecting linguistic and cultural minorities. Pan-Europa was envisaged as a synthesis of the European nation, the product of the unity of Western culture with peaceful coexistence between different traditions and linguistic-cultural minorities. Coudenhove-Kalergi's idea derives from a first attempt at intergovernmental understanding in Europe at a time when this was no more than a utopian dream (Becerril, 2016), as well as being the only possible means of maintaining its standing in the international stage. For this reason, among others, this author has been regarded as one of the «founding fathers of Europe».

The pursuit of European integration was not taken up again until after the Second World War. At the end of the war, and given the catastrophic position the continent found itself in, divided, discredited, and impoverished, there was a need to foster political rapprochement 
between the thirty sovereign states, overcoming the anarchy and incomprehension that had led to these two armed conflicts. This important objective had the enthusiastic backing of the man who had led the British Government during the Second World War, Winston Churchill, who in 1947 founded the «United European Movement» (Churchill, 2016, pp. 78-87). One year after the foundation of this movement, the event that has been called the «Congress of Europe» was held in The Hague. From 7 to 10 May 1948 this meeting, opened by Princess Juliana and by Churchill himself, was held in the Dutch city and at it the first foundations of the European Union were laid. The delegates and speakers who reached an initial agreement are regarded as the «founding fathers of Europe»: Konrad Adenauer, Paul-Henri Spaak, Jean Monnet, Alcide de Gasperi, Robert Schuman, and the aforementioned Richard Coudenhove-Kalergi ${ }^{5}$. The debates at this first congress featured disagreements between the «unionists» - mainly consisting of the British representatives, led by the Victorian Churchill- and the «federalists» including the French, Italians, Belgians, and Dutch.

At an official level, this congress's immediate achievement was the creation of the Council of Europe, whose statute was signed in London in 1949. The Council of Europe, based in Strasbourg, comprised two organisations: a Committee of Minsters and a Parliamentary Assembly. This Council played an important role in the European plan. This was because it promoted European unity based on «the principles of individual liberty, political liberty, and the pre-eminence of the law, on which true democracy is based» (Truyol, 1972, p. 35).

These principles, which were regarded as the common denominator of the shared heritage of the countries of Europe, were the foundations on which the nascent international cooperation in the old continent would be built. Indeed, thanks to the birth on 4 April 1949 of the North Atlantic Treaty Organization (NATO), and given the institutional fragility of the Council of Europe, the determined pro-Europe proposed a supranational organisation covering a smaller geographical area of European communities in the strict sense, establishing in Geneva the new «Consejo de Municipios y Regiones de Europa» in 1951.

Economically, the years following the Second World War were especially favourable. People speak of three «economic miracles» in the period between 1945 and 1963. Three states played a leading role in the growth of Western Europe: Germany, Italy, and France. Of the three «miracles», the German one was the first and most impressive. Under the leadership of the Christian Democrat Chancellor Konrad Adenauer (1945-1963), West Germany soon started to enjoy an economic boom. Thanks to strong export flows and an economy supported by a solid currency (the Deutsche Mark) it soon became one of the world's leading economic powers. This country, which had been weakened and devastated after the war, became an opulent state that was the envy of others, rising like a phoenix from the ashes. The German miracle was followed by the Italian one, which was different in nature 
and not as impressive. The northern and central regions of Italy saw the consolidation of industrial sectors such as the manufacture of automobiles and domestic appliances. After 20 years, this progress had earned Italy a strong leading position in international markets. The Italian boom was followed by the «French miracle» that saw this country grow without interruption from 1945 to $1973^{6}$. Almost thirty glorious years of unstoppable progress saw France become the hyperspecialized power in the aeronautical, agri-food, and biomedical industries that it is today.

These economic conditions would help establish a true Europe. The group of people known as the «founding fathers of Europe» are of special relevance here. Midway between integration and disassociation, the Council of Europe's activities were restricted to interesting debates since the UK had categorically opposed any loss of national sovereignty. After this false start, Jean Monnet decided that, to complete the construction of Europe, it was necessary to get used to working together in the framework of specific achievements in particular sectors. Alongside this proposal, on 9 May 1950 Robert Schuman, the French Foreign Minister -inspired by Monnet's declaration - suggested placing all of France and Germany's coal and steel production under a common high authority. The German Federal Republic, Belgium, Italy, Luxembourg, and the Netherlands backed this proposal and on 18 April 1951 - after brief negotiations - the European Coal and Steel Community (ECSC) was launched. Its founding charter was the
Treaty of Paris. With the foundation of the ECSC, the «Europe of Six» was born. For the first time its six member states ceded certain sovereign rights to a common body empowered to take care of the new body's interests. This High Authority, with supranational powers, was based in Luxembourg. The ECSC, whose first president was Monnet, operated very successfully despite the United Kingdom's refusal to join. In light of this triumph, and after the abortive attempt to establish a European Defence Community, the idea of Europe was relaunched at the meeting of the six foreign ministers of the ECSC in Messina on 1 July 1955. The boost given to the European Economic Community at this meeting has been called the relance européene. The EEC was finally established by the Treaty of Rome which was signed on 25 March 1957 and has now celebrated its seventieth anniversary. Consequently, the period between 1955 and 1957 has been referred to as the «European Spring». This agreement's aim was to guarantee free circulation of people under the protection of common citizenship and of goods by gradually erasing the national borders of the six members. The council of ministers inherited from the ECSC was joined by a «European Commission» with its headquarters in Brussels. This was an early version of a supranational power (Carpentier; Lebrun, 1994).

Naturally, creating a European common market was a strong step towards making a united Europe viable. General de Gaulle played a decisive role in this process, although, as he recalled in his memoirs, while he was not opposed to European construction, he would have fa- 
voured a Franco-German consortium. De Gaulle warned that British interference in the organisms of the EEC could open the door to American influence ${ }^{7}$. This was the main reason for the repeated refusal to allow the United Kingdom to join the Common Market, something he opposed twice (1963 and 1966). It was a matter of strengthening continental Europe. The entente cordial led by de Gaulle and Adenauer appeared to regard «consolidating» the Community as more important than "expanding» it with new members, especially if expansion could endanger the internal cohesion between these members when faced with the exterior, and in particular the USA in light of its close ties to the Commonwealth. This fact, as well as the British Empire's constant transatlantic gaze prompted de Gaulle to veto the membership of the United Kingdom. General de Gaulle proposed a «Europe of nations», in other words, a Europe of inter-state cooperation, in accordance with idea of the nation state.

Meanwhile, the repeated requests by the United Kingdom, led by the Labour prime minister Harold Wilson, to be allowed to join the Community caused intense internal discussions between the six members. In the central debate, the differences between the model of an «Atlantic Europe» with more centrifugal relationships and a «European Europe» that would prioritise the centripetal agreements of the continental line were settled. While de Gaulle opposed the United Kingdom's membership of the Community, others, and in particular the avowed supporters of supranationality Luns and Spaak backed it. Eventually after prolonged hard struggles concerning the economic questions - and helped by Churchill's absence from the political panorama - on 28 October 1971 the House of Commons approved the United Kingdom joining the Community. Following applications by two other candidates on 22 January 1972, the "Europe of Six" became the «Europe of Nine» in 1973 when the United Kingdom, Denmark and Ireland joined. This moment of expansion consolidated the second stage in European construction. The «Europe of Peoples» gave way to the "Europe of Nations», the shortfalls of which would soon become apparent. It was not until 1981, with Greece joining, that the «Europe of Ten» was discussed. In 1986, the Community expanded with the entry of Spain and Portugal, becoming the "Europe of Twelve», and so on successively until the Treaty of Maastricht in 1992 which formed the basis of the European Union (Carpentier et al., 1994; Pérez Casado, 2017).

In summary, this long and complicated history saw the laying of the first foundations of the European Union, a process featuring Richard Coudenhove-Kalergi, the pro-European Austrian author of Pan-Europa, Jean Monnet, the first President of the High Authority of the ECSC, Konrad Adenauer who was German Chancellor in the post-war period and the promotor of the German miracle, Paul Henri Spaak who was the Belgian President representing the Socialist Party, Robert Schuman who was French Foreign Minister and the founder and inspiration of the ECSC, Alcide De Gasperi of Italy who was President of the Council of Ministers, and, of course, Charles de Gaulle, 
the president of the French Fifth Republic which came into being after the colonial crisis of 1958. These people are regarded as the «founding fathers» of Europe.

\section{The pedagogical founding fa- thers of Europe}

In addition to these authors who made the construction of European unity possible on a basis of peaceful coexistence, democracy, and inter-governmental solidarity, there was a plethora of others who through pedagogical discourse accompanied this process of formation of the citizenry to consolidate these agreements. The educational traditions of the countries when the founding of the Europe of Six was founded were very different. Indeed, in the $1920 \mathrm{~s}$, after the end of the First World War, democracy had been established in some European countries while in others it was yet to be founded. Democracy was linked to three countries in particular: France, the United Kingdom, and Germany. In the first two, democracy only had to follow the long trajectory that the war had not stopped (Carpentier et al., 1994); their democratic heritage was already deeply rooted. This was not the case in Germany, where democracy was yet to be established. Its situation was different and it had initial limitations, deriving from its history. Following failed attempts at revolution in the first few months after the end of the First World War, in 1919 a Constitutional Assembly had founded a parliamentary republic defined by the Weimar Constitution that came into effect that August. The early years of this new republic were marked by clashes that underlined its political fragility. As well as successive attempted coups by the far-left and far-right, there were political assassinations, separatist movements in the Rhineland, hyperinflation in the postwar period, the Munich Putsch in 1923, led by Adolf Hitler, and the occupation of the Ruhr from 1923 to 1925 by French and Belgian troops. In contrast, from 1925 onwards the course of the nascent Republic would follow new lines. The country was stabilised under the presidency of Marshal Paul von Hindenburg who governed with a conservative majority, until he was succeeded by Hitler in 1933 .

At the same time that democracy was being introduced to these countries, in the 1920s and 1930s a wave of regimes spread across Europe, ranging from authoritarian and semi-authoritarian to the fascist extreme adopted by Mussolini in Italy after the march on Rome (1922). This wave mainly hit Mediterranean and Central Europe after a brief experience of parliamentary democracy. This was the case in Hungary under the leadership of Admiral Miklós Horthy (1920), in Austria under Monsignor Ignaz Seipel (1922), in Spain under General Primo de Rivera (1923), in Turkey under Mustafa Kemal Atatürk (1923), in Poland under Marshall Józef Pilsudski (1926), and in Portugal under António de Oliveira Salazar (1926). The Russian Revolution of 1917 and, especially, the subsequent Cold War, added to this complicated political framework. The Europe-USSR opposition during this period undoubtedly accelerated European construction, dynamizing education regarding its democratic foundations and their contrast with communism. This particular phenomenon meant 
that the first pedagogical «founding fathers of Europe» came from the democratic countries. Consequently, this first generation of pedagogues after the First World War, and affected by the events that had happened, focused their educational discourse on civic training to consolidate democracy in the state. Nonetheless, as we will see, their proposals went beyond the strictly national order and aimed connect citizens with humanity in the broader sense.

At the risk of omitting some figures, two generations of «pedagogical founding fathers» of Europe can be cited. The first generation formed the root of peaceful European construction and aimed to consolidate democracy through the autonomy of the student. This first generation, who mainly worked in the interwar period, includes Kerschensteiner, Spranger, and Natorp from Germany, Bovet, Claparède, Dottrens, and Ferrière from Geneva with their internationalist aims, Gloeckel from Austria, and the Viviani reform and Langevin-Wallon plan from the period after the Second World War in France. Once democracy had become established in these countries after the Second World War and following the establishment of the ECSC, a second generation of "pedagogical founding fathers» appeared who went beyond these early initiatives and strove to encourage continental unity through cultural exchange between their countries, giving speeches in favour of European integration through education. This is the case - with Guido Gonella, the Italian Minister of Education at the time of De Gasperi, Denis de Rougemont who was the head of the European Centre for Culture, and the definitive boost that Friedrich Schneider, a comparative educationalist, offered in his work European education of 1963. It is also worth noting a number of institutions related to these pedagogic discourses that were launched with the aim of jointly and cooperatively constructing European culture. These include the European Centre for Culture (1950) with its headquarters in Geneva and the College of Europe (1950) in Bruges, as well as the European Association of Educators, based in Darmstadt and founded in 1956.

Within this first wave of initiatives to consolidate democracy, and almost everywhere in Europe at one time or another, the ideal of state reconstruction involved establishing a unitary model of public education that had already started to be implemented before the First World War but was restarted with more enthusiasm after it. One author observes that:

The educational reform movement, which had started long before the outbreak of war, regained strength during this period, perhaps owing to the same flaws that had become apparent in the education of citizens from the belligerent countries, flaws that were attributed to shortcomings in their educational systems. Therefore, one of the first concerns of the post-war period was to reform and improve these systems (La reforma escolar en Alemania, n.d., p. 7).

As a result, educational reforms came in quick succession in the interwar period in Europe. It was noted that rebuilding intracontinental understanding would require a new model of education ${ }^{8}$. 
Consequently, it is possible to speak of the first «pedagogical founding fathers» of Europe.

We will attempt to clarify who these «pedagogical founding fathers» of Europe are to understand the role they play in forging democracy and peaceful coexistence within and between states. In reality, the long line of pedagogues who tried to establish democracy in Germany dates back to George Kerschensteiner, whose contribution formed the prelude to the educational reform that would be implemented under the Weimar Republic from 1919. According to him, educational intervention should focus on civic education with the aim of turning the democratic state into a moral community (1934, p. 33-34). This is the main mission that the public education system and education in general must undertake. It is no coincidence that changes in Germany in the years following the First World War, were based on the vocational school from Kerschensteiner's work. Public education reforms in Germany were rooted in the unified school -Einhetsschule- movement whose main supporters were the democratic and socialist parties, as well as the associations of teachers and pedagogues with liberal tendencies which included Kerschensteiner himself as well as Natorp, Fischer, and Spranger, among others. The first manifestation of this movement was in June 1914 in Kiel at the Deustche Lehvernien assembly where the association of German teachers adopted Kerschensteiner's thesis:

Under the rule of law, in other words, in a state that automatically regulates relationships between its members ac- cording to the principles of justice, public schooling creates a duty to give every child, without exception, the education they are entitled to in accordance with its provisions (Luzuriaga, 1964, p. 105).

To put this another way, a desire to establish public and democratic schools in Germany was taking shape, a project that would be delayed from the start of the First World War until the coming of the republic in 1919. Paul Natorp's ideal of Social Pedagogy, a project which also helped lay the foundations for educational democracy based on a social proposal stands out as well as Kerschensteiner's contribution, as does the work of Eduard Spranger, who argued that the aim of education was cultural pedagogy.

If pedagogy in Germany was concerned with laying the foundations of democracy owing to its lack of a parliamentary tradition, in Switzerland, with Geneva as its pedagogical capital, its interest was instead in coordinating inter-governmental mediation to re-establish peace in Europe. It is possible that the internal division of the Swiss population and the differing attachments of its citizens - some backing the French while others backed Germany - led this state to remain neutral in the First and Second World Wars. As required by the terms of the Treaty of Versailles, the League of Nations was founded in Geneva in 1920 to lead European reunification. However, it would fail in its attempts owing to the different interests of the victorious powers. Pedagogically, Geneva tried to lead educational reform by spreading the New School movement and active pedagogy. Pedagogues of the status of Ferrière, 
Claparède, Bovet, and Dottrens among others formed the Geneva School, which opted to develop experimental pedagogy without rejecting universalist ideals. Adolphe Ferrière himself ran through internationalist initiatives ranging from the Bureau International des Écoles Nouvelles (1899) and the Institut Rousseau (1912) up to the foundation of the International School of Geneva (1924-1950), and including the International League of the New School (1921). In so doing, he established a political-pedagogical theory that brought together the parameters of Europeanism, internationalism, pacifism, neutrality, intellectual cooperation, moral puritanism, and trust in education as the builder of the future society. Symbolising the spirit of Geneva, he shared the desire for a permanent peace based on disarmament, international cooperation, and the will to construct a political and educational utopia (l'Ere Nouvelle) under the principles of moral and intellectual independence and an active and central role for the child. Similarly, Claparède and Bovet were the true drivers of the Institut Rousseau. Furthermore, Bovet established an international observatory of the state of education in the world with the foundation of the Bureau International d'Education (BIE) which would become a body for documenting and promoting scientific and objective studies into educational matters. The BIE enabled the promotion and consolidation of comparative pedagogy as an epistemological, cognitive, and academic discipline (Schneider, 1966) with a clear pro-Europe mission.

These initiatives and institutions, created under the influence of the spirit of Geneva, tried to establish a European union around the principles of international understanding, collaboration, and cooperation. During the interwar period, following the break-up of the central empires, the aim was to establish peace through moral weaponry for which the New School was regarded as the most appropriate means. The active educational method was chosen to make the child an active and participatory citizen in future through self-governance and the ideal of autonomy. Ultimately, the aim was to establish a European citizenry to lead to ensure peace in the continent through active teaching and the New School movement.

The Republic of Austria was not exempt from this reforming process ${ }^{9}$ after the fall of the Austro-Hungarian Empire as a result of the First World War. In effect, the cultural, social, and economic decadence of the final years of the House of Habsburg obliged the nascent social-democratic republic to rebuild a society in the grip of a crisis. From 1919, when the socialists took power, the question of how to make Austria into a state with a new social structure in accordance with the political-social philosophy of the new republican order was considered. As part of this social transformation, which affected all areas, educational reforms were planned. To implement them Otto Gloeckel took on the role of Minister for Public Instruction (1919). Six hundred teachers associations were formed to study and practice the principles of the reform and introduce them into the Republic of Austria across all regions without exception. The two pedagogical cornerstones that sup- 
ported it were the idea of concentration, based on centres of interest, and diffusion through global learning. As can be seen, active teaching again took centre stage in establishing the republic and forming citizens. The aim was for each child to work independently. It was no coincidence that the Lernschule - school of learningwas replaced by Arbeitschule - school of work - without neglecting civic and moral education as well as ensuring religious freedom, respecting Catholic, Protestant, and Jewish schooling.

The French case is different. Under the Third Republic (1870-1940) there was a series of educational changes, from the Viviani Reform led by the pacifist prime minister René Viviani at the start of the First World War, through to the Langevin-Wallon plan (1944-1945) immediately prior to the establishment of the Fourth Republic which had Robert Schuman as president. The Viviani project (1914) was part of the reforms that introduced unitary education and extended compulsory technical elementary education and obligatory post-school training, as well as implementing the principle of free schooling and of democratising secondary and higher education, without ignoring moral and character education. This plan imposed obligatory post-school professional training for boys aged thirteen to seventeen and domestic training for girls from thirteen but only as far as sixteen. On similar lines, the Langevin-Wallon plan aimed to democratise secondary education through comprehensive schooling. Its solution was an integrated, polyvalent, and comprehensive school. This ambitious plan envisaged a school that was unitary and lay, but above all offered moral and civic education.

Alongside these Initiatives that underpinned democracy in schools, a series of notable personalities were involved in the development of the "European spirit» through pedagogy. We refer to the second generation of «pedagogical founding fathers» who, once democracy had been consolidated through education, took a step towards encouraging specifically European citizenship through educational institutions. Stefan Zweig should also be mentioned as a forebear to this generation as in 1932 he insisted on the pedagogical route to cleansing Europe of the ills of the interwar period, a total crisis that was not just economic but also epistemological, political, and philosophical, in other words, a crisis of meaning (Zweig, 2017, pp. 65-84).

In view of the obvious intra-European tensions, Guido Gonella, the Italian Minister of Education under the presidency of De Gasperi called for a return to the unity of European civilisation based on its tradition. Gonella argued for the possibilities of unity without denying plurality. His aim was not uniformity or cultural homogenisation, but rather the rediscovery of the tradition of Europe and its shared civilisation. On these lines, he said in a speech in 1961:

The European tradition is intellectual thanks to the dignity of a common way of thinking, it is legal thanks to the heritage of a shared legal system, it is spiritual thanks to the internal affinities of a common civilisation. [...] Let us then return 
to the three sources of European civilisation: Greek, Roman, and Christian. [...] The unity of Europe rebuilds itself by first restoring the intellectual and social order (Gonella, 1961, pp. 19-20) [Own translation].

The aspiration to rebuild Europe pedagogically and humanistically underlies this project. Thanks to this, Gonella can be regarded as one of the second generation of pedagogical «founding fathers of Europe». Only the cultural path, and with it the educational one, could safeguard European unity. Conscious of the violent conflicts that had overrun the continent, he expressed the urgency of espousing, through schools, active solidarity between citizens and countries. A solidarity that would be based on a new international justice that reconciled the concept of nation with that of humanity, as called for by many of the pedagogical discourses of the first generation (Gonella, 1961, p. 21). Faced with nascent anti-European fascist forces, it was necessary to seek peace through democracy rooted in an Europeanism based on patriotism.

As well as Gonella, Denis de Rougemont deserves a special mention as one of the pedagogical «founding fathers of Europe». The European Centre for Culture was launched in Geneva after the end of the Second World War, and from 1950 it was led by this convinced pro-European. It encouraged cultural exchange, emphasising what was «shared by Europe» and bringing together people and groups with similar roles. The aim of this institution was «to awaken the spirit of European solidarity in public schools, universities, and centres of popular education» (Schneider,
1963, p. 139). Supported by this centre, the AIEE was founded in 1951. This published a Bibliographie européenne with the aim of disseminating the achievements and noting problems relating to the unification of Europe.

Rougemont's impetus was decisive for the pedagogical integration of Europe. As director of the European Centre for Culture he undertook a great deal of work training an elite group of pro-Europe politicians and educators. He was aware that a customs union was not enough to achieve a united Europe, and from the founding moment he called for a continental political, social, cultural, and economic federation. In his writing he underlined the importance he gives education for this inter-government understanding.

To say that everything depends on education is to say that everything depends on educators and their training. The future of the united Europe is at stake in teacher training centres. Until there is an antinationalist and pro-European shift in them, and until the effects of this change are felt in the secondary education of our countries, the very foundations of the union will seem to have developed in step with the demands of politicians and economists. And so before «making Europe» we have to «make ourselves European». And this fundamentally happens in spirits: without a prior «cultural revolution» there can be no revolution in the socio-political institutions, or it will not really get started (Rougement, 1975, p. 72) [Own translation].

In short, beyond the Europe of markets, Rougemont emphasised the need 
for a Europe of citizens. It was necessary to forge a European citizenry through education. First of all by training teachers in this way at teacher training colleges, so that, like a chain reaction, this change would be subsequently felt in primary and secondary schools in each member state. Rougemont was aware of the need for a «cultural revolution» to strengthen the European spirit. Education alone, and no other possible means, was the instrument for achieving European unification in the minds of citizens. People had to come to idea of Europe through education, with a sense of pro-European citizenship awakening in each person, freeing them from the chains of nationalism that had led to the two intracontinental wars.

Nonetheless, the decisive push for European education came from Friedrich Schneider, who can be regarded as another of the pedagogical «founding fathers» of Europe. He argued for the need for a discipline called European education which as well as being the title of one of his works, was also a curriculum proposal.

The aim of European education was none other than to ensure this European heritage -we call it European civilisation or the European spirit- and make it known as much as possible to many of the members of peoples of Europe; in other words, to form a European feeling in them, a European conscience, is an eminently important task at present. Therefore, as well as political and economic integration, a cultural integration is also required to consolidate these two types (Schneider, 1961, p. 56).
As Rougemont had previously stressed, the European tradition had to be supported through education, that is, training European citizens. Accordingly, he proposed an educational curriculum that contained, among other aspects, a history of pedagogy in the European sense. His intention was, as his predecessors has already stated, to encourage Europeanism through patriotism, always respecting national ties. This is the form a European education had to take; its sense of cohesion could not be at odds with the national community but instead had to be compatible with it. To this end, Schneider examined European educational systems, emphasising what they had in common. Their common features included: the duration of the period of compulsory education (this varied from six to ten years and was an achievement of the first generation of pedagogical founding fathers), the belief in continuous education (beyond primary level and professional training education in European countries continues throughout life), and, finally, education had a tripartite structure in almost all countries with primary, secondary, and higher education. If educational problems, as he himself would find, were shared, the solution also had to be shared by all of the member states of this union. Therefore, pedagogical research also had to be supranational. Ultimately, all efforts should be focussed on seeking out the European spirit, both with regards to its problems and its solutions, neither more nor less.

\section{In conclusion}

Having seen this overview of the origins of Europe, there is no doubt that 
efforts have been made since its earliest days to express it as a pedagogical project (Vilanou, Prats, Longares, 2016, p. 17). In accordance with the cultural and spiritual dimension of the old continent, Europe has been an educational reference model, from the German and Swiss proposals to the Austrian and French ones in favour of constructing a supranational state of peaceful coexistence and citizen solidarity. Nonetheless, it is now at a crossroads. In the year of the sixtieth anniversary of its establishment it faces the break-up of its unity with the exit of the United Kingdom and the start of the so-called Brexit process with its possible impacts on the Eurozone. Anticipating its possible decline, Pérez Casado (2017) affirms that faced with the threat of the emerging countries and of its most powerful competitor, especially since the electoral triumph of Donald Trump, the alternative is a bigger and better Europe. It is necessary to rethink it, avoiding technocratic pitfalls and a generalised loss of confidence in its institutions and returning the central role to its citizens. On the same lines, he maintains that «these in-depth reforms require the construction of a democratic pedagogy that involves citizens in its objectives, making them partners in a new stage of European construction» (2017, p. 33). Consequently, the reestablishment of the united Europe or the European Union, respecting and returning to the original principles of c0operation, will go through the educational route. As Jean Monnet would lament: «If I had to do it again, I would begin with culture». Today, more than ever, it makes sense to reread the pedagogical «founding fathers of Europe» who introduced dem- ocratic pedagogy and European citizenship, conditions that make integration possible.

In this new Europe that we now find - post-industrial, postmodern, post organic, and multicultural- pedagogical discourse must follow new paths to confront the challenges that affect European reconciliation, achieved during this 100-year journey since the First World War. Therefore, if Europe is one thing now, or will be, it involves the pedagogical and educational project. Javier Solana notes that the European Union is the most innovative and successful process of integration in the history of humankind $(2015$, p. 9). If we wish to maintain the levels of shared prosperity as well as the freedom and equality of citizens, European integration is vital. Peace, prosperity, the rule of law, respect for difference, solidarity, liberty, democracy, and social justice are the values that today shape European identity and are our «common heritage». As such we must protect them and they must be strengthened through education. Today more than ever, Europe is a pedagogical project.

\section{Notes}

The research this article is based on was made possible by RecerCaixa.

Voyenne notes that Wilson, an avid reader of Kant, dreamt of the «philosophical project of perpetual peacen. The League of Nations, inspired by Wilson, comprised the triumph of law over Germany's aggressive attempts at establishing hegemony in Europe. In other words, the victory of democracy, justice and peace over Germany's ambitions for military dominance in Europe (1965, p. 165).

Prior to the attempts at European construction or reconstruction, after the First World War, there 
had been a series of works known as literature of crisis. One example of this is The decline of the West by Oswald Spengler, who in 1918 denounced the crisis of linear evolutionism and of the pro. gressive designs of social development (Esquirol, 1994, p. 34).

4 Coudenhove-Kalergi (1923). Paneuropa. In Truyol (1972), pp. 81-83. Paneuropa was republished in 2010 in Madrid by Encuentro.

5 Voyenne has stated with regards to this congress: «For the first time, all of the men who were deter. mined to build Europe were together. This resulted in an atmosphere of exceptional enthusiasm. How. ever, after this first contact, it could be seen that two trends, at least, seriously divided the European forces. On the one hand, the unionists remained as solidly opposed to anything that could limit the sov. ereignty of the states as they had previously been. [...]. On the other hand, the federalists, called for an effective federation, with its own powers») (1965, p. 188).

6 De Gaulle in his memoirs recounts the successive investments made in France during the period from 1958 to 1962 in trade, agriculture, and electronics, as well as aeronautics and industries relating to gas. All of these processes of modernisation gave a strong boost to the French regions that special. ised in certain sectors (1970, pp.183-184).

7 De Gaulle himself in his memoirs recalls the difficulties of building a European Europe faced with the threat of the Atlantic Trojan Horse that the ear. ly integration of Britain would have involved (1970, pp. 202-203).

8 It should be no surprise that Comparative Edu. cation became established as an academic discipline in this period. As Schneider notes, «at the end of the First World War, several circumstances favoured the development of the comparative sci. ence of education. States with developed school systems felt the need to submit them to a rigor. ous assessment; the new states saw education as the essential means for their foundation and consolidation. And, above all, the desire to achieve civic democratic opinions and humanity led them to concern themselves with educational plans») (1966, p. 46).

9 On the Austrian educational reform, Luzuriaga writes: «The First World War was to stop this de. velopment of the new public school and only with the end of the war could it be pursued. Accord. ingly, the second stage, the 'federal educational institutions' in Vienna were true 'new schools' but these were public and national in character») (2002, p. 53).

\section{References}

Anonymous (n.d.). La reforma escolar en Alemania. Madrid: Ediciones de La Lectura-Espasa Calpe. Aguirre, M. (2017). Salto al vacío. Crisis y declive de los Estados Unidos. Barcelona: Icaria.

Adenauer, K. (2014). El final del nacionalismo. Madrid: Encuentro.

Becerril, B. (2016). Estudio introductorio Europe Unite. En W. Churchill, Europa Unida. Dieciocho discursos y una carta (pp. 5-45). Madrid: Encuentro.

Cañellas, A., Solé, J., Pallach, J., Reventós, J., Trias, R. y Pujol, J. (1975). Les terceres vies a Europa. Barcelona: Nova Terra.

Carpentier, J., Lebrun, F. (1994). Breve Historia de Europa. Madrid: Alianza.

Coudenhove-Kalergi, R. (2010). Pan-Europa. Madrid: Encuentro.

De Gaulle, C. (1970). Memorias de esperanza. La renovación (1958-1962). Madrid: Taurus.

De Gasperi, A. (2011). Europa, escritos y discursos. Madrid: Encuentro.

De Habsburgo, O. (2011). El camino de Europa. Madrid: Encuentro.

De Madariaga, S. (1951). Bosquejo de Europa. México: Hermes.

Esquirol, J. M. (1994). D’Europa als homes. Barcelona: Cruïlla.

Ferro, M. (2014). La Gran Guerra (1914-1918). Madrid: Alianza.

Fichte, J. (1968). Discursos a la nación alemana. Madrid: Taurus.

Filho, L. (1936). La escuela nueva. Barcelona: Labor.

Freinet, C. (1975). El nacimiento de una pedagogía popular. Barcelona: Laia. 
Fullat, O. (2005). L'Autèntic origen dels europeus. El cristianisme en la formació d'Occident. Barcelona: Pòrtic.

Gonella, G. (1961) Unitat d'Europa. Barcelona: Rafael Dalmau.

Güell, A. (Coord.) (1998). El Dilema de Europa. Barcelona: Ediciones del Bronce.

Howard, M. (2012). La Primera Guerra Mundial. Barcelona: Crítica.

Kamen, H. (1986). La sociedad europea (15001700). Madrid: Alianza.

Kerschensteiner, G. (1928). El alma del educador y el problema de la formación del maestro. Barcelona: Labor.

Kerschensteiner, G. (1934). La educación cívica. Barcelona: Labor.

Kerschensteiner, G. (n.d.). Concepto de escuela del trabajo. Madrid: La Lectura.

Luzuriaga, L. (1964). La escuela pública democrática. Buenos Aires: Losada

Luzuriaga, L. (2002). La escuela nueva pública. Madrid: Losada.

Martín, V. (2015). Europa, un salto a lo desconocido. Madrid: Encuentro.

Natorp, P. (1915). Curso de Pedagogía. Madrid: La Lectura.

Negrín, O. \& Vergara, J. (2003). Teorías e Instituciones contemporáneas de educación. Madrid: Centro de Estudios Ramón Areces.

Pérez Casado, R. (2017). La Unión Europea. Historia de un éxito tras las catástrofes del siglo $X X$. Valencia: Publicacions de la Universitat de València.

Rougemont, D. (1975). ;Europeos, uníos! Barcelona: Aymá.

Schneider, F. (1966). La Pedagogía Comparada. Barcelona: Herder.

Schneider, F. (1963). Educación europea. Barcelona: Herder.

Solana, J. (2015). Prólogo. In V. Martín, Europa, un salto a lo desconocido (pp. 9-10). Madrid: Encuentro.
Soler, J. (2008). Entre Europa i Amèrica Llatina: Adolf Ferrière i l'Escola Nova a Barcelona. Temps d'Educació, 35, 229-248.

Truyol, A. (1972). La integración europea. Madrid: Tecnos.

Vilanou, C., Prat, E., \& Longares L. (2016). Entre la crónica i el compromís de futur. Temps d'Educació, 50, 11-29.

Voyenne, B. (1965). Historia de la idea europea. Barcelona: Labor.

Witte, E. \& Backheuser, E. (1933). La escuela única. Barcelona: Labor.

Zweig, S (2017). La desintoxicación moral de Europa y otros escritos políticos. Barcelona: Plataforma editorial.

\section{Author biographies}

Isabel Vilafranca Manguán has a Phd in Pedagogy from the Universidad de Barcelona. She is an Associate Professor in the Department of Theory and History of Education in the Faculty of Education at the Universidad de Barcelona. She specialises in studying pedagogical ideas and theories, in particular the philosophical and educational currents relating to German idealism. She currently leads the project «Pedagogical thinking and educational discourses in European construction a hundred years after the First World War. Between the past and the future» as part of the RecerCaixa programme.

Raquel Cercós Raichs has a degree in Pedagogy from the Universidad de Barcelona and is an Associate Professor in the Universidad de Barcelona's Faculty of Education. She has focussed on studying the concepts, discourses, and narratives of Pedagogies of Masculinity through the links established between power, gender, and education, with her area of attention 
being Victorian England and its reception Theory and History of Education in the and critique in the United States. Anoth- Faculty of Education at the Universidad er of her lines of research is corporality de Barcelona and is an Advisor on the Soand pedagogy. cial Education degree at the Universitat

Jordi Garcia Farrero won the Spe- Oberta de Catalunya (UOC). He is the aucial Doctorate Prize from the Universi- thor of the book Caminar. Experiencias $y$ dad de Barcelona (2012-2013). He is an prácticas formativas (Walking: Training Assistant Professor in the Department of experiences and practices, 2014). 


\section{revista española de pedagogía año LXXVI, n 270, mayo-agosto 2018 \\ Spanish Journal of Pedagogy \\ year LXXVI, n. 270, May-August 2018}

\section{Table of Contents}

\section{Studies}

Xavier Úcar

Metaphors for socio-educational intervention:

pedagogical implications for practice

209

Inmaculada Asensio Muñoz, Elvira Carpintero Molina,

Eva Expósito Casas and Esther López Martín

How much gold is in the sand? Data mining

with Spain's PISA 2015 results

225

Francisco José Fernández-Cruz, Ma José Fernández-

Díaz and Jesús Miguel Rodriguez Mantilla

Design and validation of an instrument

to measure teacher training profiles in

information and communication technologies

\section{Notes}

María Teresa Caro Valverde, María Isabel de VicenteYagüe Jara and Maria Teresa Valverde González Teacher perception of methodological habits for informal argumentation in text commentary
Ana Rodriguez-Meirinhos and Esther Ciria-Barreiro

Review of interventions to improve pragmatic language skills in children with behaviour and attention problems 295

Pilar Pineda-Herrero, Anna Ciraso-Cali and Mary Armijos-Yambay

Employability and competences of Pedagogy,

Psychology, and Educational Psychology

graduates: A comparative study of employers and graduates

Isabel Vilafranca Manguán,

Raquel Cercós Raichs and Jordi García Farrero

The pedagogical founding fathers of Europe:

247 foundational education discourses

for European integration, one hundred

years after the First World War

Jonathan Ruiz-Jaramillo and

Antonio Vargas-Yáñez

Teaching structures on Architecture degrees. ICT.

273 based methodology and teaching innovation 


\section{Book reviews}

Gargallo López, B. (Coord.) Enseñanza centrada

en el aprendizaje y diseño por competencias en la

Universidad. Fundamentación, procedimientos

y evidencias de aplicación e investigación

[Learning.centred teaching and designing for

skills in the university: foundations, procedures,

and evidence for application to research]

(Vicent Gozálvez). Ballester, L. \& Colom, A.

Epistemologías de la complejidad y educación

[Epistemologies of complexity and education]
(Carlos Alberto Pabón Meneses).

Monarca, H. \& Thoilliez, B. (Coords.)

La profesionalización docente: debates

y propuestas [The professionalisation

of teaching: debates and proposals]

(Francisco Esteban Bara).

Balduzzi, E. Narrazione educativa e generatività

del perdono [Educational narration and the

creation of forgiveness] (Mauricio Bicocca).

373

Call for papers

403

This is the English version of the research articles and book reviews published originally in the Spanish printed version of issue 270 of the revista española de pedagogía. The full Spanish version of this issue can also be found on the journal's website http://revistadepedagogia.org.

ISSN: 0034-9461 (Print), 2174-0909 (Online)

https://revistadepedagogia.org/

Depósito legal: M. 6.020 - 1958

INDUSTRIA GRÁFICA ANZOS, S.L. Fuenlabrada - Madrid 